\title{
LETTERS
}

\section{Don't freeze specialists out of guideline development}

In an article published in CMAJ, ${ }^{1}$ Drs. Jatoi and Sah level serious accusations against specialist physicians' professionalism, integrity and commitment to care, which the Canadian Psychiatric Association believes are unfounded and demand a response.

The authors suggest that "independent [...] organizations that have few or no vested interests in the medical services at stake" ${ }^{1}$ are better placed to develop guidelines, given that those formulated by specialists are troubled by specialty bias, fee-for-service conflicts of interest or both, and specialists' interests may not always align with those of the public.

Organizations such as the Canadian Task Force on Preventive Health Care (CTFPHC) are funded by governments, which, as payors, have a clear interest in controlling budgets and reducing costs. Physicians are bound by codes of ethics that do not apply to politicians and functionaries. The simplistic conclusion that specialist physicians recommend more interventions and care because they make more money doing so is shockingly unscientific and denigrating, and ignores the fact that specialists have been trained and are qualified to make certain clinical decisions.

Psychiatrists are specialist physicians with extensive medical training in the causes, diagnosis, treatment and ongoing care of mental disorders in patients of all ages. Psychiatrists' medical training and expertise in psychological development allow them to understand the interaction between the physical, social and psychological aspects of mental disorders. This training permits psychiatrists to prescribe medication appropriately, provide psychotherapeutic treatments and work with patients, especially those with chronic or episodic conditions, to improve their quality of life. Often, part of the treatment or rehabilitation plan will include referral to or collaboration with a range of social and support services, as well as with patients' families.

Guidelines developed by nonspecialists and that are based solely on clinical trial data may oversimplify treatment and ignore clinical scenarios that require comprehensive judgment in addition to data, and may be harmful to patients. Data cited in guidelines are from research done by specialist clinicians working in the field. If these clinicians are biased, how are the data not biased from the very beginning?

The Canadian Psychiatric Association has recently expressed concerns about standards developed by governmentfunded organizations that appear to exclude specialist physicians systematically, or are formulated without a meaningful consultation process. ${ }^{2,3}$ Too often, "consultation" may consist of a request for input and a subsequent rejection of recommendations and even references. Formal objections may be ignored, or discredited by insinuations of financial bias.

It is arbitrary and irresponsible to develop standards for health care without the participation of specialist physicians, given their deep knowledge about patients' needs and key role in delivering care. It is difficult to imagine standards for airline safety being developed without input from pilots and aerospace engineers, yet more and more this is what seems to be happening with respect to health care.

Canada's psychiatrists are committed to providing evidence-based care for people with mental illnesses. The Canadian Psychiatric Association will continue to advocate for policies and guidelines that are based on the best research evidence and allow for the best possible outcomes for Canadians.

\section{Wei-Yi Song MD}

Psychiatrist, president, Canadian

Psychiatric Association, Victoria, BC

Cite as: CMAJ 2019 September 9; 191:E1008. doi: 10.1503/cmaj. 72772

\section{References}

1. Jatoi I, Sah S. Clinical practice guidelines and the overuse of health care services: need for reform. CMAJ 2019;191: E297-8.

2. Sinha N. "it is arbitrary and irresponsible to develop standards for mental health care without the participation of psychiatrists" - @DrWeiYiSong, @ CPA_APC President speaks up about a serious breach of trust in Canadian healthcare: http://bit.ly/2P3Q4Ib \#mentalhealth \#CDNhealth [Tweet]. 2018 Nov. 1. Available: https://twitter.com/nachiketa_sinha/ status/1058074159924817920 (accessed 2019 May 15).

3. Sinha N. The stigma is still alive as disregard for experts continues in Canadian mental healthcare. I Am Me! Not My Illness! 2018 Nov. 3. Available: https:// iammenotmyillness.com/blog/the-stigma-is-still -alive-as-disregard-for-experts-continues-in-canadian -mental-healthcare (accessed 2019 May 15).

Competing interests: Wei-Yi Song is president of the Canadian Psychiatric Association and was a member of the Canadian Network for Mood and Anxiety Treatments Depression Work Group, which developed the CANMAT 2016 Clinical Guidelines for the Management of Adults with Major Depressive Disorder. 